\title{
Control of mosquito larvae in catch basins using pyriproxyfen and the mechanism underlying residual efficacy
}

\author{
Kazunori OHASHI* \\ * Corresponding author: ohashik@sc.sumitomo-chem.co.jp \\ Health and Crop Sciences Research Laboratory, Sumitomo Chemical Co., Ltd., \\ Takatsukasa 4-2-1, Takarazuka, Hyogo 665-8555, Japan
}

(Received: 26 June 2017; Accepted: 14 November 2017)

\begin{abstract}
Storm water catch basins are a major source of dengue vector mosquito larvae (Diptera: Culicidae) in the urban and residential areas of Japan. In this study, the mechanisms underlying the residual efficacy of the insect growth regulator pyriproxyfen in treated catch basins were examined. One tablet of the formulation of pyriproxyfen, SumiLarv ${ }^{\mathbb{R}}$ Tablet, was applied to a catch basin at 1-month intervals in Takarazuka, Japan. Simultaneously, one disk of the matrix-release formulation of pyriproxyfen, SumiLarv ${ }^{\circledR} 2 M R$, was applied to another catch basin. Weekly bioassays of water sampled from the treated basins showed insufficient efficacy against fourth-instar larvae of laboratory-reared Aedes albopictus. However, over 6 months, mortality rates greater than 95\% and 100\% were observed in pupae collected from the basins treated with SumiLarv ${ }^{\mathbb{R}}$ Tablet and SumiLarv ${ }^{\mathbb{R}} 2 \mathrm{MR}$, respectively. Laboratory experiments were conducted to clarify the difference in the efficacy between the water sampling method and the pupal collection method. Bioassay using water samples containing added antibiotics indicated that biodegradation of the active ingredient in water caused the low observed efficacy when using the water sampling method. By contrast, the active ingredient that was retained by organic matter persisted for several weeks and was gradually released to the water. In addition, larval ingestion of organic matter that had absorbed the active ingredient appeared to enhance mortality rates. Thus, organic matter in the water was responsible for the high efficacy of the pupal collection method. This study confirmed the importance of organic matter as a reservoir of pyriproxyfen in water.
\end{abstract}

Key words: dengue, vector control, larval source management, larvicide, residual efficacy, chemical persistence

\section{INTRODUCTION}

In recent years, disease-vector mosquitoes in Japan have been subject to limited control actions, but the necessity for vector control was recognized after an outbreak of dengue fever in Tokyo during September 2014. Although it was necessary to spray insecticide to kill infective mosquitoes during the outbreak, it is advised to focus on managing larval sources during non-outbreak periods, to reduce the density of vector populations (World Health Organization, 2009). Aedes albopictus (Skuse) was the vector mosquito that transmitted the dengue virus during the outbreak in Tokyo (Tsuda et al., 2015). Because large numbers of the immature stages of this species are found in storm water catch basins installed in the drainage systems of urban and residential areas (Kawada et al., 2010; Tsuda, 2012), these structures should be targeted for larval source management. The World Health Organization (WHO) recommends the elimination of larval habitats wherever possible and suggests the application of larvicides to larval sources that cannot be removed (WHO, 2009). For example, Ocampo et al. (2014) identified the importance of street catch basins as Aedes breeding sites in Colombia, and found that a monthly application of larvicide caused a reduction in the incidence of dengue. In Japan, it is currently permitted to use several types of insecticides, including organophosphates, pyrethroids, and insect growth regulators (IGRs), for control of mosquito larvae. Of these, IGRs have often been applied in catch basins because of their safety to humans and ease of application (e.g., Ogata, 2013; Seki et al., 2015).

The IGR pyriproxyfen is a juvenile hormone analog with extremely low toxicity to mammals (Food and Agricultural Organization of the United Nations, 1999), whereas extremely low doses of the chemical delivered to mosquito larval habitats inhibit adult emergence (Hirano et al., 1998). Therefore, WHO has recommended the use of pyriproxyfen to control mosquito larvae (WHO, 2001). Although pyriproxyfen is non- or low-toxic to larval stages, except at very high dosages, it does cause pupal mortality, and at low doses induces abnormal adult emergence (Estrada and Mulla, 1986; Mulla and Darwazeh, 1988). The period during which mosquitoes are sensitive to pyriproxyfen occurs before and immediately after pupation (Iwanaga and Kanda, 1988; Schaefer et al., 1988; Kono et al., 1997). The $50 \%$ lethal concentrations $\left(\mathrm{LC}_{50}\right)$ of pyriproxyfen have been reported to be $0.043,0.023$, and $0.0046 \mathrm{ppb}$ against Anopheles stephensi Liston, Aedes aegypti (L.), and Culex pipiens pallens Coquillett, 
respectively (Hatakoshi et al., 1987). Although pyriproxyfen is characterized by long residual activities (Kawada et al., 1988; Schaefer et al., 1988; Takagi et al., 1995; Nayar et al., 2002; Vythilingam et al., 2005; Ritchie et al., 2013), the duration of efficacy is thought to be affected by many factors, including decomposition of the active ingredient in water and adsorption onto organic matter, soil, or containers (Mulligan and Schaefer, 1990; Miyamoto et al., 1993; Suman et al., 2013). For example, the half-life of pyriproxyfen has been reported to be 1.6 days in natural pond water (Miyamoto et al., 1993) but it was extended to 7.5 days or 10 days on organic matter or in sediments, respectively (Schaefer et al., 1991; Miyamoto et al., 1993). These observations suggest that the chemical presence on organic matter and sediments can be key factors affecting the residual efficacy of this insecticide.

In this study, I investigated how organic matter affects the residual efficacy of pyriproxyfen by applying it in catch basins that are naturally inhabited by mosquito larvae. First, the dose-mortality response of pyriproxyfen was examined in Ae. albopictus. Second, the residual efficacy of the insecticide against mosquito larvae was evaluated using two different methods: exposure of laboratory-reared larvae to treated water and collection of pupae from treated habitats. Third, laboratory experiments were conducted to clarify how organic matter influences the residual efficacy of pyriproxyfen.

\section{Materials and Methods}

\section{Insecticides}

Technical grade pyriproxyfen (Sumitomo Chemical Co., Ltd., Tokyo, Japan); a tablet formulation containing $\quad 0.5 \%$ pyriproxyfen, SumiLarv ${ }^{\circledR}$ Tablet SES (SC Environmental Science Co., Ltd., Osaka, Japan); and a long-lasting matrix-release formulation containing $2 \%$ active ingredient, SumiLarv ${ }^{\mathbb{B}} 2 \mathrm{MR}$ (Sumitomo Chemical Co., Ltd., Tokyo, Japan) were used for the experiments. The SumiLarv ${ }^{\mathbb{B}}$ Tablet disperses the active ingredient in water within a few minutes with the help of carbon dioxide, whereas the slow-release formulation SumiLarv ${ }^{\circledR} 2 \mathrm{MR}$ has sustained efficacy for at least 6 months (Ohashi and Shono, 2015). SumiLarv ${ }^{\circledR} 2 \mathrm{MR}$ was recommended by the WHO in 2017 for the control of mosquito larvae.
Dose-mortality response of pyriproxyfen

A $10 \mathrm{~mL}$ stock solution of $1000 \mathrm{ppm}(0.1 \% \mathrm{w} / \mathrm{v})$ pyriproxyfen was prepared by weighing $10 \mathrm{mg}$ of technical grade pyriproxyfen into a $20 \mathrm{~mL}$ vial and adding $10 \mathrm{~mL}$ acetone. The stock solution was then serially diluted to prepare test solutions. Batches of 20 late fourth-instar larvae of a laboratory-reared susceptible strain of Ae. albopictus were introduced into $200 \mathrm{~mL}$ glass beakers containing $200 \mathrm{~mL}$ of deionized water. Small or damaged larvae were replaced. Three replicates (in total 60 larvae) were set up for each concentration and untreated control. A $200 \mu \mathrm{L}$ of test solution was added to $200 \mathrm{~mL}$ of water (i.e., 1000-fold dilution) using a micropipette. The final concentration ranged from 0.04 to $0.0025 \mathrm{ppb}$ which includes the concentration causing 50\% inhibition of emergence. In the untreated control, $200 \mu \mathrm{L}$ of acetone was added to the water. The water was mixed well immediately after adding the solution using a disposable spatula. The larvae were provided with a small amount of larval food, a 1:1 mixture of powdered animal food (CE-2; CLEA Japan Inc., Tokyo) and dried yeast (Ebios ${ }^{\circledR}$; Mitsubishi Tanabe Pharma Co., Osaka), which was added to the water daily. The beakers were maintained at $26 \pm 1^{\circ} \mathrm{C}$, $60 \% \pm 10 \%$ relative humidity $(\mathrm{RH})$, and a photoperiod of $15 \mathrm{~h}: 9 \mathrm{~h}$ (L:D). After 3 days exposure, both dead and surviving individuals that had not pupated were removed from the water using a disposable pipette. The beakers were covered with a nylon mesh and pupae were allowed develop until adult emergence. After a further 3 days, the number of successfully emerged adults was counted. Adults that did not emerge from pupal cases were considered dead. The results for the three replicates of each concentration were pooled for calculation of the percentage adult emergence based on the number of individuals that had pupated after exposure for 3 days. The test was repeated three times using fresh test solutions and different batches of larvae. The percentage inhibition of adult emergence for each concentration was calculated as follows (WHO, 2005):

$$
\text { Emergence inhibition }(\%)=100-(\mathrm{T} \times 100 / \mathrm{C}) \text {, }
$$

where $\mathrm{T}=$ percentage emergence in treated batches and $\mathrm{C}=$ percentage emergence in the untreated batches.

Treatment in the field

To examine residual efficacy of pyriproxyfen at

Table 1. Number of mosquitoes collected in three catch basins before treatment and their emergence rates.

\begin{tabular}{lccccc}
\hline \hline \multirow{2}{*}{ Catch basin used for: } & \multirow{2}{*}{$\begin{array}{c}\text { No. of } \\
\text { pupae collected }\end{array}$} & Culex pipiens & Aedes albopictus & Aedes japonicus & \% Emergence \\
\cline { 3 - 5 } & 13 & 11 & 1 & 0 & 92.3 \\
SumiLarv Tablet & 20 & 12 & 8 & 0 & 100 \\
SumiLarv 2MR & 7 & 0 & 3 & 4 & 100 \\
Control & & & & 0 \\
\hline
\end{tabular}




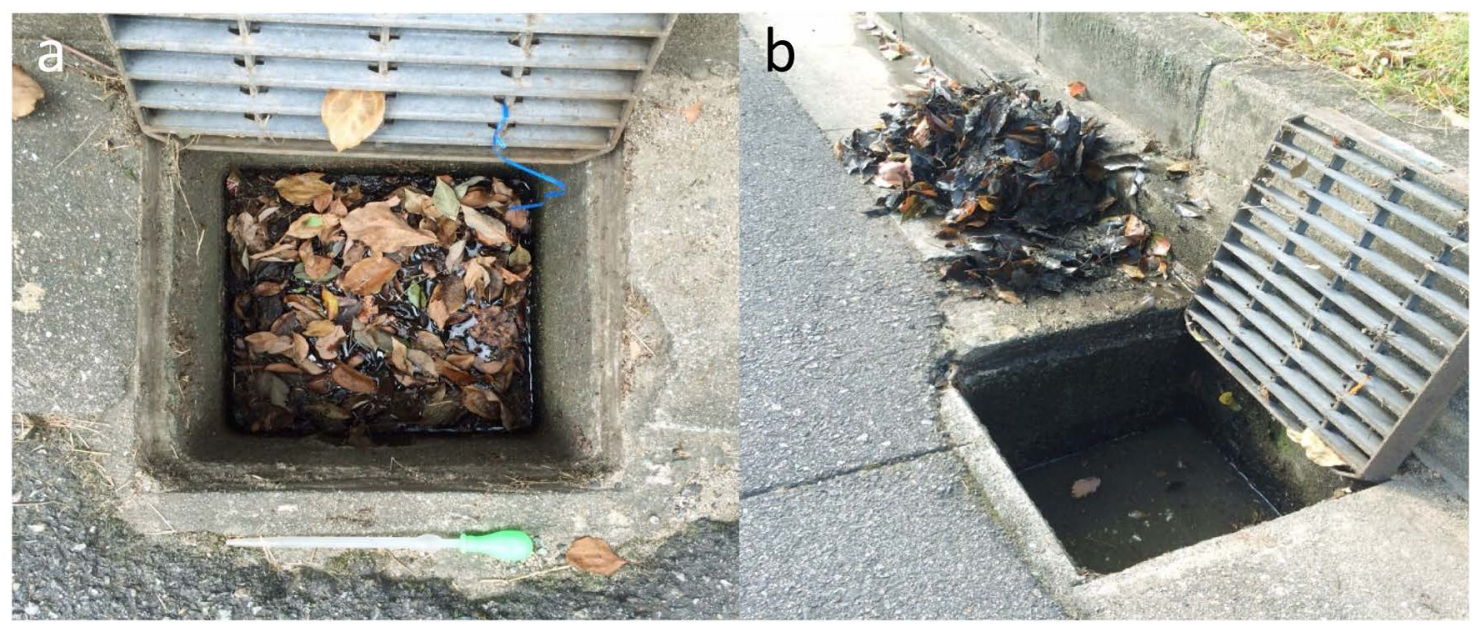

Fig. 1. (a) A catch basin containing fallen leaves; (b) Removal of the leaves on October 27, 2016.

the following experiments, the treated water was prepared in the field condition. Three catch basins located at the Health and Crop Sciences Research Laboratory in Takarazuka, Japan, which permanently hold water and are populated by mosquito larvae, were selected at random. One basin was treated with the SumiLarv ${ }^{\circledR}$ Tablet, one was treated with SumiLarv ${ }^{\circledR} 2 \mathrm{MR}$, and one was left as an untreated control. Each catch basin was $36 \times 36 \mathrm{~cm}$ in size, with a water depth of $14 \mathrm{~cm}$, giving a total water volume of 18.1 L. During a baseline survey conducted on May 29, 2016, the pupae of Cx. pipiens, Ae. albopictus, and Ae. japonicus (Theobald) were found in the catch basins (Table 1). The percentages of emergence in collected pupae were greater than 92.3\% (Table 1). The basins contained soil on the bottom, and leaves that had fallen from Cinnamomum camphora (L.) Sieb. were present on the water surface (Fig. 1a). SumiLarv ${ }^{\circledR}$ Tablet was applied at a rate of one tablet $(0.5 \mathrm{~g})$ per month from June to November, except July (dates of application were June 11, August 4, September 4, October 4, November 1, and November $26,2016)$. To assess the slow-release of pyriproxyfen, one disk $(1.95 \mathrm{~g})$ of SumiLarv ${ }^{\circledR} 2 \mathrm{MR}$ was placed in the second catch basin on June 11, 2016, and was fixed in place with string. Fallen leaves were allowed to remain in the basins until October 27, 2016, when all the leaves were occasionally removed by a gardener (Fig. 1b). The amounts of fallen leaves sometimes decreased after heavy rain.

\section{Biological evaluation of the treated water}

The residual efficacy of pyriproxyfen was evaluated using two methods. In the water sampling method, $500 \mathrm{~mL}$ water was collected using a plastic beaker $(2 \mathrm{~L})$ once a week from all the three basins described above. Immediately after collection, the water samples were left in $900 \mathrm{~mL}$ glass bottles for $1 \mathrm{~h}$ to allow the particles of organic matter to settle. Thereafter, $50 \mathrm{~mL}$ of supernatant water was placed in each of four
$150 \mathrm{~mL}$ aluminum cups, and 10 late fourth-instar larvae of a laboratory-reared susceptible strain of Ae. albopictus were released into each container, which was subsequently covered with a lid. The larvae were fed a larval food as described above and were maintained in a room controlled at $26 \pm 1^{\circ} \mathrm{C}$. The number of adults was recorded after all adults had either emerged or pupae had died and mortality rates were calculated. Adults that did not successfully emerge from pupal cases were considered dead.

In the pupal collection method, a maximum of 25 pupae (mean: 10.8 $\pm 6.4 \mathrm{SD}$ ) and $50 \mathrm{~mL}$ water samples were collected by dipping from the treated and untreated catch basins once a week. The collection dates were sometime delayed due to rain. Both the pupae and water were placed in a clean $200 \mathrm{~mL}$ cup and covered with a lid. The number of emerged adults was recorded after either all adults had emerged or pupae had died. The evaluation were carried out between June and December 2016, with the exception of July.

\section{Meteorological data}

The meteorological data (precipitation and daily mean air temperature) were obtained from an online database of the Japan Meteorological Agency (www. jma.go.jp). The data were recorded at the nearest weather station (Toyonaka) from our study site, the distance is ca. $7 \mathrm{~km}$.

\section{Persistence of the active ingredient in water}

The effect of organic matter on decomposition of the active ingredient was examined based on biological efficacy. Two liters of test water was sampled using a plastic beaker from the SumiLarv ${ }^{\circledR}$ Tablet-treated and untreated-control catch basins. Immediately after collection, the water samples were allowed to settle in glass bottles for $1 \mathrm{~h}$, and $250 \mathrm{~mL}$ of supernatant water was placed in each of four $500 \mathrm{~mL}$ glass beakers per basin. One set of two beakers was subject to bioassay 
without additives. In a second pair of beakers, five fallen leaves of $C$. camphora (3-4g per beaker) collected from each catch basin were immersed into the test water after rinsing in deionized water. A batch of 25 laboratory-reared late fourth-instar Ae. albopictus larvae was released into the test water, maintained at $26 \pm 1^{\circ} \mathrm{C}$, and fed $15 \mathrm{mg}$ of larval food. To evaluate short-term decomposition of the active ingredient, newly pupated individuals were removed at $24 \mathrm{~h}$ intervals and were transferred to separate $150 \mathrm{~mL}$ aluminum-lidded cups. The rate of adult emergence was recorded and used to calculate mortality rate for each pupation date.

A further experiment was conducted to examine whether decomposition of active ingredient in water is caused by bacteria. One liter of test water was sampled from the basin treated with SumiLarv ${ }^{\circledR}$ Tablet and the untreated control catch basin, allowed to settle in glass bottles for $1 \mathrm{~h}$, and then $100 \mathrm{~mL}$ of supernatant water was placed in each of six $200 \mathrm{~mL}$ glass beakers per basin. One set of three replicate beakers was directly assessed. A second set of three beakers was assessed after adding antibiotics (penicillin $100 \mathrm{units} / \mathrm{mL}$ and streptomycin $100 \mu \mathrm{g} /$ $\mathrm{mL}$; Wako Pure Chemical Industries, Ltd.) to each beaker. A batch of 20 laboratory-reared late fourthinstar Ae. albopictus larvae was released in the water at $26 \pm 1^{\circ} \mathrm{C}$ and fed $5 \mathrm{mg}$ of larval food. Pupae were removed at $24 \mathrm{~h}$ intervals and transferred to separate $150 \mathrm{~mL}$ aluminum-lidded cups. The mortality rate for each pupation date was calculated. All bioassays were started on the day when the water was sampled.

Effect of organic matter on the residual efficacy

The effect on residual efficacy of organic matter that had absorbed the active ingredient was evaluated using fallen leaves immersed in pure water. Three liters of deionized water was placed in a $10 \mathrm{~L}$ stainlesssteel container and 10 fallen leaves $(8 \mathrm{~g})$ that had been collected from the SumiLarv ${ }^{\circledR}$ Tablet-treated catch basin were rinsed and placed in the water (Fig. 2). A batch of 20 late fourth-instar Ae. albopictus larvae was released in the water without any restriction of movement (Fig. 2), and provided with $30 \mathrm{mg}$ of larval food. Simultaneously, 10 larvae were released into a 9-cm-diameter floating cage constructed of a stainlesssteel net suspended at the level of the water surface to isolate larvae from the leaves (Fig. 2), and were fed several grains of animal food (CE-2; CLEA Japan Inc., Tokyo). A further 10 larvae were maintained in a floating cage containing a fallen leaf $(0.8 \mathrm{~g})$ collected from the same catch basin (Fig. 2). A separate $10 \mathrm{~L}$ beaker containing $3 \mathrm{~L}$ deionized water and one floating cage but without fallen leaves was used as an untreated control. Resulting pupae were transferred to $150 \mathrm{~mL}$ aluminum-lidded cups to record mortality rates. As all larvae pupated within 1 week, the experiment was

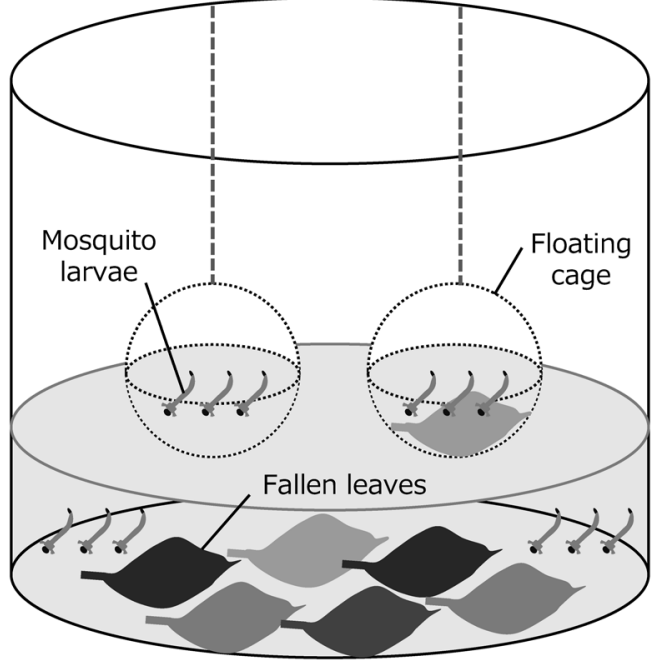

Fig. 2. Diagram of a $10 \mathrm{~L}$ stainless-steel container holding $3 \mathrm{~L}$ of deionized water and 10 fallen leaves collected from the catch basin treated with the SumiLarv ${ }^{\circledR}$ Tablet. Two floating cages containing 10 fourth-instar larvae of a susceptible strain of Aedes albopictus with and without fallen leaves, respectively, were suspended at the level of the water surface. Another 20 larvae were released outside the floating cages.

repeated five times every 7 days using a new batch of larvae.

Persistence of the active ingredient in larvae

The persistence of the active ingredient in larval bodies was examined using 106 fourth-instar $C x$. pipiens pallens larvae collected on November 7, 2016, from the catch basin treated with SumiLarv ${ }^{\circledR} 2 M R$. Larvae were rinsed with fresh water and maintained in a plastic tray containing $2 \mathrm{~L}$ seasoned tap water and were fed powdered larval food. Resulting pupae were then transferred at $24 \mathrm{~h}$ intervals to separate $150 \mathrm{~mL}$ aluminum lidded cups to calculate the mortality rate for each pupation date. Emerged adults were identified based on their male genitalia (Ohashi et al., 2014).

\section{Statistical analysis}

Concentrations of pyriproxyfen causing $50 \%$ and 99.9\% inhibition of adult emergence $\left(\mathrm{IE}_{50}\right.$ and $\left.\mathrm{IE}_{99.9}\right)$ with $95 \%$ confidence intervals were calculated by using log-probit regression analysis. A generalized liner model was used to analyze the effects of the treatments on pupal mortality in each laboratory experiment. The proportion of dead individuals was fitted with a binomial distribution with logit link function. Presence of fallen leaves, addition of antibiotics, and treatment arms with or without floating cages were the factors used in each model. Time (days or weeks) after treatment and interactions between treatment and time were also included in each model. The analyses were performed with JMP11.2.1 (SAS Institute Japan Inc., Tokyo, Japan). 


\section{Results}

Dose-mortality response of pyriproxyfen

The relationship between pyriproxyfen concentration and percentage emergence inhibition is shown in Fig. 3. $\mathrm{IE}_{50}$ and $\mathrm{IE}_{99.9}$ values were determined as $0.009 \mathrm{ppb}(95 \% \mathrm{CI}: 0.008-0.010 \mathrm{ppb})$ and $0.15 \mathrm{ppb}$ (0.112-0.210 ppb), respectively.

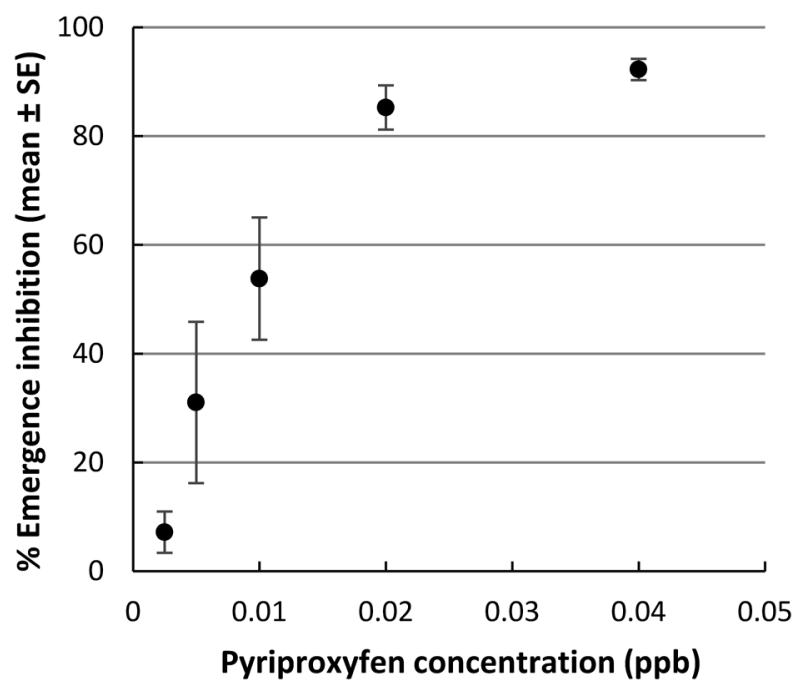

Fig. 3. Relationship between pyriproxyfen concentration and emergence inhibition rates in Aedes albopictus.
Biological efficacy in the field test

Meteorological data for the duration of the field test and mortality rates based on the water sampling method in the catch basins treated with SumiLarv ${ }^{\circledR}$ Tablet and SumiLarv ${ }^{\circledR} 2$ MR are shown in Fig. 4a. Under the SumiLarv ${ }^{\circledR}$ Tablet treatment, 65\%-100\% mortality was observed 1 week after each treatment was implemented, with rates decreasing gradually up to 4 weeks after treatment (Fig. 4a). In some weeks, mortality rates were higher than those of the previous week. For SumiLarv ${ }^{\mathbb{B}} 2 \mathrm{MR}$, mortality rates were maintained at $>63 \%$ throughout the test periods, but rarely reached $100 \%$ (Fig. 4a). Mortality rates in the untreated control were $<20 \%$. No obvious relationship between rainfall and mortality rates was observed for either formulation.

The mortality rates based on the pupal collection method are presented in Fig. 4b. The pupae collected from the catch basin treated with SumiLarv ${ }^{\circledR}$ Tablet showed $>95 \%$ mortality rates during the test period, with the exception of a dip to $71 \%$ at 3 weeks after the first treatment (Fig. 4b). Treatment with SumiLarv ${ }^{\circledR} 2$ MR produced $100 \%$ mortality at all the observation points, except on dates when pupae were not collected (Fig. 4b). Mortality rates in the untreated control were $<25 \%$ with the exception of one record of $57 \%$. Neither rainfall nor the removal of fallen leaves seemed to affect the mortality rates in either treatment.
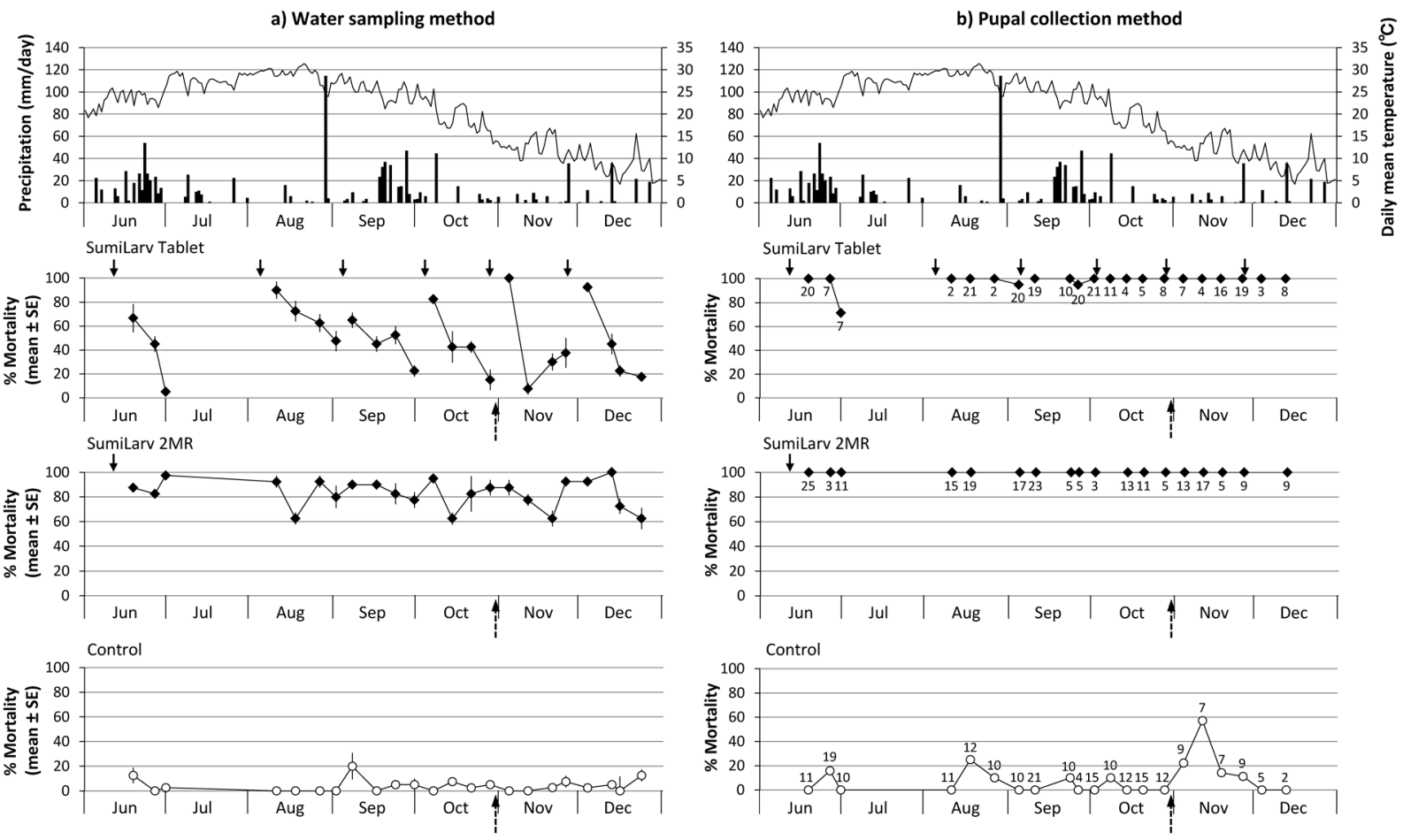

Fig. 4. Residual activity of SumiLarv ${ }^{\circledR}$ Tablet and SumiLarv ${ }^{\circledR} 2 M R$ in catch basins determined using (a) bioassays using water sampled from the treated catch basins and laboratory-reared fourth-instar larvae of Aedes albopictus, and (b) direct observation of pupae collected from the treated catch basins. The upper panel shows daily rainfall (bar) and daily mean air temperature (line) recorded at the nearest meteorological station (Toyonaka, Japan). Solid arrows indicate the date of treatment. The dotted arrow indicates the date when fallen leaves were removed. Numbers represent the number of pupae observed. 
No pupae were collected after mid-December.

Effect of organic matter and bacteria on biological efficacy

A mortality rate of $83 \%$ in individuals that pupated at 2 days after exposure was observed in water sampled from the treated catch basin without fallen leaves (Fig. 5a). This rate fell to $0 \%$ at 3 days after exposure. However, $100 \%$ mortality was observed in individuals placed in water containing fallen leaves at 2 days or longer after exposure (Fig. 5a). Statistical analysis indicated that mortality was significantly affected by the presence of fallen leaves $\left(\chi^{2}=21.8\right.$; $\mathrm{df}=1 ; P<0.0001)$ and changed significantly over time $\left(\chi^{2}=9.49 ; \mathrm{df}=1 ; P=0.0021\right)$. Moreover, there was a significant interaction between treatment and time $\left(\chi^{2}=17.8 ; \quad \mathrm{df}=1 ; \quad P<0.0001\right)$. Similarly, individuals that pupated in the treated water without antibiotics showed a $12 \%$ mortality rate at 3 days after exposure, versus a $100 \%$ morality rate at 2 days or longer after exposure in water containing antibiotics (Fig. 5b). Mortality was significantly affected by the addition of antibiotics $\left(\chi^{2}=14.0 ; \mathrm{df}=1 ; P=0.0002\right)$ and changed significantly over time $\left(\chi^{2}=4.42 ; \mathrm{df}=1 ; P=0.035\right)$, and there was a significant interaction between treatment and time $\left(\chi^{2}=38.7 ; \mathrm{df}=1 ; P<0.0001\right)$.

\section{Effect of organic matter on the residual efficacy}

The mortality rates in deionized water containing 10 fallen leaves collected from the catch basin treated with SumiLarv ${ }^{\circledR}$ Tablet are shown in Fig. 6. In the control water without fallen leaves, the mortality rates were $<5 \%$ (data not shown). In the treatment with fallen leaves, for larvae outside the floating cages, there was $100 \%$ mortality at 0 weeks post-treatment, and $>70 \%$ mortality at 4 weeks. In comparison, the mortality rates of larvae isolated from the fallen leaves by the floating cages were lower than those of larvae outside the cages. The mortality rates of the larvae maintained in the floating cage with fallen leaves were high, and comparable to those of larvae outside the cage (Fig. 6 ), indicating that contact with the fallen leaves was the main factor contributing to the higher morality. Mortality rates in the container with fallen leaves were significantly different among three treatment arms $\left(\chi^{2}=40.5 ; \mathrm{df}=2 ; P<0.0001\right)$, and declined significantly over time $\left(\chi^{2}=31.8 ; \mathrm{df}=1 ; P<0.0001\right)$; however, there was no significant interaction between treatment and
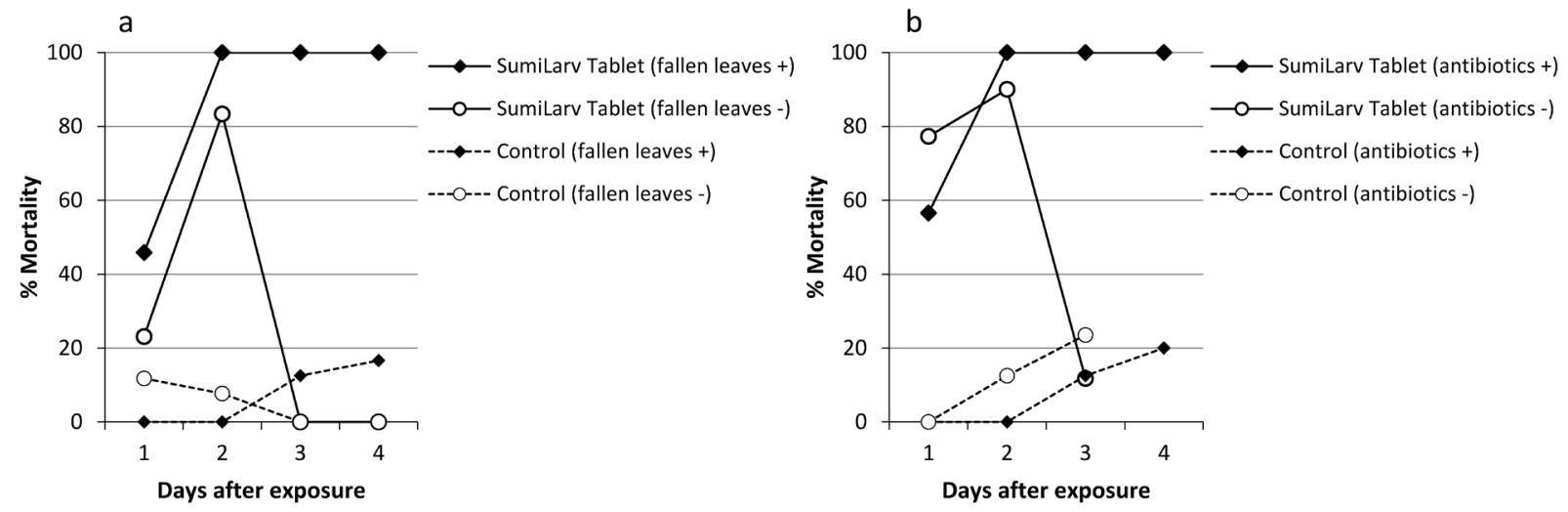

Fig. 5. Pupal mortality of laboratory-reared fourth instar larvae of Aedes albopictus on each pupation date after exposure to test water sampled from the catch basin treated with SumiLarv ${ }^{\mathbb{B}}$ Tablet. Treated water was tested (a) with and without five fallen leaves collected from the same catch basin and (b) with and without antibiotics (penicillin-streptomycin solution).

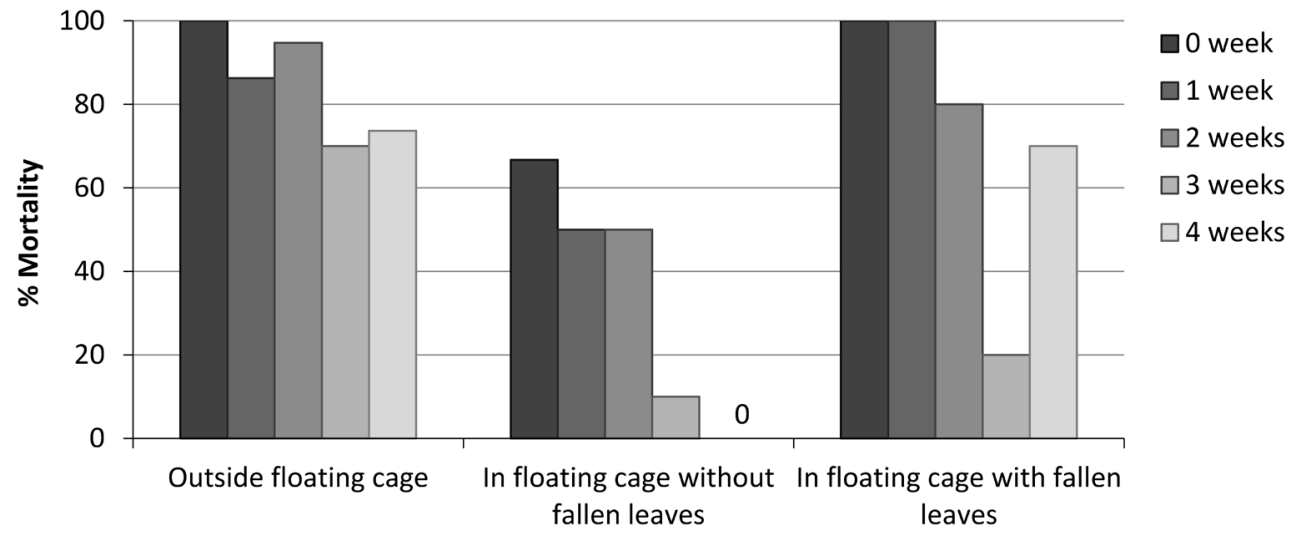

Fig. 6. Residual activity of pyriproxyfen on fallen leaves collected from the catch basin treated with the SumiLarv ${ }^{\circledR}$ Tablet. Leaves were immersed in deionized water and laboratory-reared fourth-instar larvae of Aedes albopictus were released into the water once a week. Mortality of larvae inside and outside the floating cages, with and without fallen leaves, was observed for 5 weeks. 


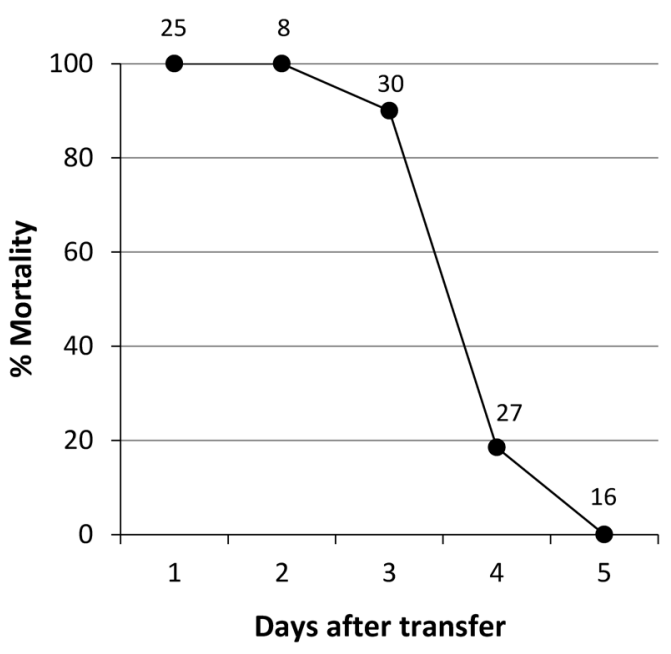

Fig. 7. Mortality of Culex pipiens pallens larvae on each pupation date after transfer from the catch basin treated with SumiLarv ${ }^{\circledR} 2$ MR to clean water. Numbers indicate the sample size.

time $\left(\chi^{2}=0.83 ; \mathrm{df}=2 ; P=0.66\right)$.

Persistence of the active ingredient in larvae

The pupal mortality of larvae collected from the catch basin treated with SumiLarv ${ }^{\circledR} 2 M R$ and transferred to clean water is shown in Fig. 7. Individuals pupating up to 3 days after transfer showed $>90 \%$ mortality, whereas the mortality rate of those pupating at 4 days or more after transfer decreased to $<19 \%$.

\section{Discussion}

Bioassays performed in the field test indicated that the observed efficacy of pyriproxyfen varies depending on the evaluation method. The water sampling method, in which test water was sampled from treated catch basins, appeared to show low efficacy, whereas the pupal collection method, in which naturally occurring pupae were examined, showed high efficacy. As the pupal collection method is expected to reflect the actual efficacy in the catch basin, mortality rates based on the water sampling method would be underestimated. On the basis of the pupal collection method, the SumiLarv ${ }^{\circledR}$ Tablet and SumiLarv ${ }^{\circledR} 2 \mathrm{MR}$ were effective for 4 weeks and at least 6 months, respectively. Under the SumiLarv ${ }^{\circledR}$ Tablet treatment, the mortality rate, based on the pupal collection method, decreased to $71 \%$ at 3 weeks after the first treatment in June, probably because the active ingredient was flushed out with a large amount of rainwater during the rainy season. However, mortality rates greater than $95 \%$ were maintained in the second and subsequent treatments, and the rate did not decrease, even when there were large quantities of rain and when the fallen leaves were removed. Therefore, the SumiLarv ${ }^{\circledR}$ Tablet would be expected to have sufficient efficacy when used at a rate of one tablet per catch basin per month.

The SumiLarv ${ }^{\circledR} 2 \mathrm{MR}$ application completely inhibited the emergence of adults from pupae collected from the catch basin for at least 6 months (i.e., June to mid-December), when pupae were no longer collected due to the onset of winter. The SumiLarv ${ }^{\circledR} 2 M R$ application led to higher mortality rates than the SumiLarv ${ }^{\circledR}$ Tablet by both evaluation methods. Rainwater and the removal of fallen leaves hardly affected the results. Therefore, a single application of the formulation would cover the entire mosquito breeding season.

The factors leading to the apparent low efficacy with the water sampling method may include the decomposition of the active ingredient after sampling the water. The emergence of adults was not inhibited among individuals that pupated at 3 days after treatment in the test water without fallen leaves or antibiotics, which could be attributed to the fact that the active ingredient in the water decreased from an effective level above $0.04 \mathrm{ppb}$ to an ineffective level below $0.0025 \mathrm{ppb}$ within 3 days. The period of sensitivity to pyriproxyfen is limited to around the time of pupation (Iwanaga and Kanda, 1988; Schaefer et al., 1988; Kono et al., 1997), and thus the active ingredient could have had a weaker effect on individuals that pupated later. Because mortality rates did not decrease in the test water containing antibiotics, biodegradation by bacteria could be responsible for the low efficacy observed with the water sampling method. Kawada et al. (1988) investigated the residual efficacy of pyriproxyfen under field conditions using an open concrete pot, and reported that efficacy was greater among larvae exposed to the test water in a floating cage in situ, compared with larvae treated in the test water brought to the laboratory. This observation was probably attributable to biodegradation of the active ingredient.

Even with the water sampling method, mortality rates were not decreased by the presence of fallen leaves collected from the SumiLarv ${ }^{\circledR}$ Tablet-treated catch basin. This indicates that the active ingredient had been absorbed by the fallen leaves and affected the larvae. The clean water with the leaves from the treated catch basin partly inhibited the adult emergence in the floating cage in the laboratory experiment. This result demonstrates that the active ingredient absorbed by the fallen leaves was released to the water. In addition, higher mortality rates were observed in the larvae that were not separated from the leaves than in larvae isolated from the leaves in floating cages. Thus, contact with or ingestion of treated organic material enhances the efficacy of pyriproxyfen. As Ae. albopictus larvae feed on organic matter in their aquatic habitat (Dieng et al., 2002), they could have ingested a large amount of the active ingredient through consumption of fallen 
leaf material.

The emergence of adult Cx. pipiens pallens after larvae were collected from the SumiLarv ${ }^{\circledR} 2 M R$ treated catch basin was inhibited, even when they were transferred to clean water. Thus, these larvae had already retained the active ingredient in their bodies, presumably through ingesting organic matter. In comparison, the adult emergence of individuals that pupated at 4 days or more after transfer was not inhibited, suggesting that the active ingredient was metabolized or egested within 4 days, and did not affect them during their period of sensitivity. Miyamoto et al. (1993) reported that the half-life of pyriproxyfen in Cx. pipiens pallens larvae after $48 \mathrm{~h}$ exposure to treated water at a concentration of $10 \mathrm{ppb}$ was 0.13 days. Thus, our results appear to indicate a longer persistence of the chemical in mosquito larvae than reported earlier. Pyriproxyfen ingested through consumption of organic matter might persist in larvae longer than when the chemical is taken up via water.

Mulligan and Schaefer (1990) reported that a longer residual efficacy of pyriproxyfen in lagoons was observed in polluted sources. In the current study, pyriproxyfen persisted on organic matter for several weeks. This result is consistent with analysis of Schaefer et al. (1991), who reported a 7.5-day halflife of pyriproxyfen on organic matter. These findings support the suggestion that organic matter contributes to the long-term residual activity of pyriproxyfen in water. In conclusion, the high residual efficacy of pyriproxyfen following application in mosquito larval habitats is achieved because the active ingredient is (1) retained in organic matter, such as fallen leaves, (2) released gradually from the organic matter to the water, and (3) ingested when larvae feed on organic matter.

\section{ACKNOWLEDGements}

I thank N. Sakamoto for providing technical assistance with the experiments and Y. Shono and two anonymous reviewers for critical reading on this manuscript. I also thank SC Environmental Science Co., Ltd., Osaka, Japan and Sumika Technoservice Corporation, Hyogo, Japan for providing the SumiLarv ${ }^{\circledR}$ Tablet sample and the mosquito larvae, respectively.

\section{REFERENCES}

Dieng, H., Mwandawiro, C., Boots, M., Morales, R., Satho, T., Tuno, N., Tsuda, Y. and Takagi, M. 2002. Leaf litter decay process and the growth performance of Aedes albopictus larvae (Diptera: Culicidae). J. Vector Ecol., 27: 31-38.

Estrada, J. G. and Mulla, M. S. 1986. Evaluation of two new insect growth regulators against mosquitoes in the laboratory. J. Am. Mosq. Control Assoc., 2: 57-60.

Food and Agricultural Organization of the United Nations (FAO). 1999. Pesticide residues in food-1999. Report of the Joint Meeting of the FAO Panel of Experts on Pesticide Residues in
Food and the Environment and the WHO Core Assessment Group. (FAO Plant Production and Protection Paper 153), 293 pp., Rome, Italy.

Hatakoshi, M., Kawada, H., Nishida, S., Kishida, H. and Nakayama, I. 1987. Laboratory evaluation of 2-[1-methyl-2-(4phenoxyphenoxy)-ethoxy] pyridine against larvae of mosquitoes and housefly. Jpn. J. Sanit. Zool., 38: 271-274.

Hirano, M., Hatakoshi, M., Kawada, H. and Takimoto, Y. 1998. Pyriproxyfen and other juvenile hormone analogues. Rev. Toxicol., 2: 357-394.

Iwanaga, K. and Kanda, T. 1988. The effects of a juvenile hormone active oxime ether compound on the metamorphosis and reproduction of an anopheline vector, Anopheles balabacensis (Diptera: Culicidae). Appl. Entomol. Zool., 23: 186-193.

Kawada, H., Dohara, K. and Shinjo, G. 1988. Laboratory and field evaluation of an insect growth regulator, 4-phenoxyphenyl (RS)2-(2-pyridyloxy) propyl ether, as a mosquito larvicide. Jpn. J. Sanit. Zool., 39: 339-346.

Kawada, H., Maekawa, Y., Abe, M., Ohashi, K., Ohba, S. and Takagi, M. 2010. Spatial distribution and pyrethroid susceptibility of mosquito larvae collected from catch basins in parks in Nagasaki city, Nagasaki, Japan. Jpn. J. Infect. Dis., 63: 19-24.

Miyamoto, J., Hirano, M., Takimoto, Y. and Hatakoshi, M. 1993. Insect growth regulators for pest control, with emphasis on juvenile hormone analogs. Present status and future prospects. In: Pest Control with Enhanced Environmental Safety (ACS symposium series 524) (ed. Duke, S. O., Menn, J. J. and Plimmer, J. R.), pp. 144-168. American Chemical Society, Washington, DC. Kono, Y., Iwabuchi, K. and Takahashi, M. 1997. Changes in susceptibility to pyriproxyfen, a JH mimic, during late larval and early pupal stages of Culex pipiens molestus. Med. Entomol. Zool., 48: 85-89.

Mulla, M. S. and Darwazeh, H. A. 1988. Efficacy of new insect growth regulators against mosquito larvae in dairy wastewater lagoons. J. Am. Mosq. Control Assoc., 4: 322-325.

Mulligan, F. S. III and Schaefer, C. H. 1990. Efficacy of a juvenile hormone mimic, pyriproxyfen (S-31183), for mosquito control in dairy wastewater lagoons. J. Am. Mosq. Control Assoc., 6: 89-92.

Nayar, J., Ali, A. and Zaim, M. 2002. Effectiveness and residual activity comparison of granular formulations of insect growth regulators pyriproxyfen and s-methoprene against Florida mosquitoes in laboratory and outdoor conditions. J. Am. Mosq. Control Assoc., 18: 196-201.

Ocampo, C. B., Mina, N. J., Carabali, M., Alexander, N. and Osorio, L. 2014. Reduction in dengue cases observed during mass control of Aedes (Stegomyia) in street catch basins in an endemic urban area in Colombia. Acta Trop., 132: 15-22.

Ogata, K. 2013. Survey of Aedes albopictus (Skuse) in a residential area in Yokohama and its control. Pestology, 28: 89-99 (In Japanese with English abstract).

Ohashi, K. and Shono, Y. 2015. Recent progress in the research and development of new products for malaria and dengue vector control. Sumitomo Kagaku, 2015: 4-14 (In Japanese with English version). [accessed June 26, 2017] Available from: https://www. sumitomo-chem.co.jp/english/rd/report/theses/docs/2015E_1. pdf.

Ohashi, K., Tsuda, Y., Kasai, S., Kawada, H. and Takagi, M. 2014. Hybridization between sympatric populations of Culex pipiens pallens and Culex pipiens f. molestus (Diptera: Culicidae) in Nagasaki, Japan. Med. Entomol. Zool., 65: 67-72.

Ritchie, S. A., Paton, C., Buhagiar, T., Webb, G. A. and Jovic, V. 2013. Residual treatment of Aedes aegypti (Diptera: Culicidae) in containers using pyriproxyfen slow-release granules (Sumilarv 0.5G). J. Med. Entomol., 50: 1169-1172.

Schaefer, C. H., Dupras, E. F. Jr. and Mulligan, F. S. III. 1991. Studies on the environmental persistence of S-31183 (pyriproxyfen): Adsorption onto organic matter and potential for leaching 
through soil. Ecotoxicol. Environ. Saf., 21: 207-214.

Schaefer, C. H., Miura, T., Dupras, E. F. Jr., Mulligan, F. S. III and Wilder, W. H. 1988. Efficacy, nontarget effects, and chemical persistence of S-31183, a promising mosquito (Diptera: Culicidae) control agent. J. Econ. Entomol., 81: 1648-1655.

Seki, N., Iwashita, Y., Moto, R., Kamiya, N., Kurita, M., Tahara, N., Hasegawa, M., Shinkai, T., Hayashi, Y., Sadamasu, K., Kai, A., Nakajima, Y., Watase, H., Ueda, T., Maeda, H., Kobayashi, K., Ishizaki, Y. and Hiromatsu, K. 2015. An autochthonous outbreak of dengue type 1 in Tokyo, Japan 2014. Nippon Koshu Eisei Zasshi, 62: 238-250 (In Japanese with English abstract).

Suman, D. S., Wang, Y., Dong, L. and Gaugler, R. 2013. Effects of larval habitat substrate on pyriproxyfen efficacy against Aedes albopictus (Diptera: Culicidae). J. Med. Entomol., 50: 1261-1266.

Takagi, M., Tsuda, Y. and Wada, Y. 1995. Evaluation of effective period of a juvenile hormone mimic, pyriproxyfen, against Aedes albopictus: Preliminary experiments in the laboratory and the field. Trop. Med. (Nagasaki), 37: 87-91.

Tsuda, Y. 2012. Ecology of mosquitoes inhabiting a park in urban Tokyo, Japan: Seasonal prevalence of larvae occurred in catch basins. Med. Entomol. Zool., 63: 95-101 (In Japanese with English abstract).

Tsuda, Y., Maekawa, Y., Ogawa, K., Itokawa, K., Komagata, O., Sasaki, T., Isawa, H., Tomita, T. and Sawabe, K. 2015. Biting density and distribution of Aedes albopictus during the September 2014 outbreak of dengue fever in Yoyogi Park and the vicinity in Tokyo Metropolis, Japan. Jpn. J. Infect. Dis., 69: 1-5.

Vythilingam, I., Luz, B. M., Hanni, R., Tan, S. B. and Tan, C. H. 2005. Laboratory and field evaluation of the insect growth regulator pyriproxyfen (Sumilarv 0.5G) against dengue vectors. J. Am. Mosq. Control Assoc., 21: 296-300.

World Health Organization (WHO). 2001. Report of the Fourth WHOPES Working Group Meeting, WHO/CDS/ WHOPES/2001.2, 102 pp., WHO, Geneva.

World Health Organization (WHO). 2005. Guidelines for Laboratory and Field Testing of Mosquito Larvicides, WHO/ CDS/WHOPES/GCDPP/2005.13, 39 pp., WHO, Geneva.

World Health Organization (WHO). 2009. Dengue: Guidelines for Diagnosis, Treatment, Prevention and Control-New edition. WHO/HTM/NTD/DEN/2009.1, 147 pp., WHO, Geneva. 